\title{
Research Paper: Curcumin Effects on Memory Impairment and Restoration of Irregular Neuronal Distribution in the Hippocampal CA1 Region After Global Cerebral Ischemia in Male Rats
}

\author{
Leila Kamali Dolatabadi ${ }^{1}$ (D), Masoumeh Emamghoreishi ${ }^{1,2,3^{*}}$ (D), Mohammad Reza Namavar ${ }^{4,5,6}$, Hamze Badeli Sarkala $^{6}$ (it)
}

1. Department of Neuroscience, School of Advanced Medical Sciences and Technologies, Shiraz University of Medical Sciences, Shiraz, Iran

2. Department of Pharmacology, School of Medicine, Shiraz University of Medical Sciences, Shiraz, Iran.

3. Research Center for Psychiatry and Behavior Science, Shiraz University of Medical Sciences, Shiraz, Iran.

4. Clinical Neurology Research Center, Shiraz University of Medical Sciences, Shiraz, Iran

5. Histomorphometry and Stereology Research Center, Shiraz University of Medical Sciences, Shiraz, Iran

6. Department of Anatomy, School of Medicine, Shiraz University of Medical Sciences, Shiraz, Iran.

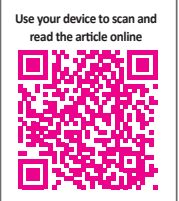

Citration: Kamali Dolatabadi, L., Emamghoreishi, M., Namavar, MR., \& Badeli Sarkala, H. Curcumin Effects on Memory Impairment and Restoration of Irregular Neuronal Distribution in the Hippocampal CA1 Region After Global Cerebral Ischemia in Male Rats. (2019). Basic and Clinical Neuroscience, 10(5), 527-540. http://dx.doi.org/10.32598/bcn.9.10.365

http://dx.doi.org/10.32598/ben.9.10.365

Article info:

Received: 12 Mar 2018

First Revision: 25 Apr 2018

Accepted: 03 Oct 2018

Available Online: 01 Sep 2019

Keywords:

Curcumin, Global cerebral ischemia, Memory, Neurons

\begin{abstract}
AB S T RACT
Introduction: Global Cerebral Ischemia (GCI) causes neuronal damage with subsequent neurological and cognitive impairments. Curcumin has anti-inflammatory, antioxidant, and neuroprotective properties, which makes it a potential candidate for improving GCI-induced impairments. This study aimed to investigate the effects of curcumin on the neurological and memory deficits, as well as spatial neuronal distribution in the Cornu Ammonis 1 region after GCI in rats.

Methods: 56 Sprague-Dawley male rats were randomly assigned into 4 groups of sham $(n=14)$, control ( $\mathrm{n}=14)$, curcumin $50 \mathrm{mg} / \mathrm{kg}(\mathrm{n}=14)$, and curcumin $100 \mathrm{mg} / \mathrm{kg}(\mathrm{n}=14)$. Each group was divided into the two subgroups of short-term ( 7 days) and long-term ( 28 days) treatment periods. The Neurological Severity Score (NSS), passive avoidance task, and the traction test were performed at postoperative days of $0,1,2,3,7,14,21$, and 28 . The novel object recognition test and Voronoi tessellation were carried out on days 7 and 28 after GCI.

Results: Curcumin $100 \mathrm{mg} / \mathrm{kg}$ significantly decreased neurological severity score on postoperative days of 7 and 28 compared with the control $(\mathrm{P}<0.001)$ and curcumin $50 \mathrm{mg} / \mathrm{kg}$ groups $(\mathrm{P}<0.05-\mathrm{P}<0.001)$, respectively. Also, curcumin $100 \mathrm{mg} / \mathrm{kg}$ significantly increased step-through latency times on postoperative days of 3-28 and 14-28 compared with the control $(\mathrm{P}<0.05-\mathrm{P}<0.001)$ and curcumin $50 \mathrm{mg} / \mathrm{kg}$ groups $(\mathrm{P}<0.01-\mathrm{P}<0.001)$. Moreover, it increased the novelty preference index during the novel object recognition test in the 28-day treatment subgroup after GCI. Curcumin $(100 \mathrm{mg} / \mathrm{kg})$ could maintain the neuronal aggregation in the CA1 region after GCI at a level near to what is generally observed in normal rats.

Conclusion: Curcumin could improve memory and neurological deficits and restore irregular neuronal distribution in the CA1 region after GCI in a time-dependent manner, and its higher dose was more effective than its lower dose. Curcumin may have beneficial effects on reducing brain complications after ischemia.
\end{abstract}

* Corresponding Author:

Masoumeh Emamghoreishi, PhD.

Address: Department of Pathology, Pharmacology, Parasitology, School of Medicine, Shiraz University of Medical Sciences, Shiraz, Iran. Tel: +98 (71) 32307591

E-mail:emamm@sums.ac.ir 


\section{Highlights}

- Curcumin can be a potential candidate for improving memory and neurological problems after global cerebral ischemia.

- A high dose of curcumin $\neg(100 \neg \mathrm{mg} / \mathrm{kg})$ and its long-term administration (28 days) significantly decreased neurological severity score after global cerebral ischemia.

- A high dose of curcumin $\neg(100 \neg \mathrm{mg} / \mathrm{kg}$ ) and its long-term administration (28 days) significantly increased stepthrough latency time under passive avoidance task and the novelty preference index during the novel object recognition after global cerebral ischemia.

- A high dose of curcumin $\neg(100 \neg \mathrm{mg} / \mathrm{kg}$ ) and its long-term administration (28 days) could maintain the neuronal aggregation in the CA1 hippocampal region after global cerebral ischemia.

\section{Plain Language Summary}

Transient Global Cerebral Ischemia (GCI) is a cerebrovascular condition dramatically reducing cerebral blood flow leading to a selective and delayed pyramidal neuronal death in the hippocampal CA1 area. Herbal medications and their active ingredients are a potential source for finding new remedies to improve cognitive and neurological deficits that occur following GCI. Several studies supported the anti-inflammatory, antioxidant, and neuroprotective effects of curcumin as an active constituent of turmeric. Because of its biological properties, curcumin is an appropriate candidate for improving injuries after brain ischemia. This study is an attempt to assess the acute and chronic effects of curcumin on the spatial neuronal distribution in CA1 area and on the neurological symptoms and memory impairment after GCI. Experiments were conducted on 56 adult male Sprague Dawley rats. Based on the results, a high dose of curcumin $(100 \mathrm{mg} / \mathrm{kg}$ ) and its long-term administration (28 days) showed a greater effect on memory function, neurological deficits, and regular neuronal aggregation in the CA1 hippocampal region after GCI.

\section{Introduction}

rain ischemia is a major cause of disability B and the second leading cause of mortality worldwide (Li, Shalabi, Ji, \& Meng, 2017). Transient Global Cerebral Ischemia (GCI) is a cerebrovascular condition dramatically reducing cerebral blood flow leading to a selective and delayed pyramidal neuronal death in the hippocampal Cornu Ammonis 1 area (Kiryk et al., 2011). Hippocampal damage is associated with cognitive impairments observed in rodents and humans following GCI (Gulinello, Lebesgue, Jover-Mengual, Zukin, \& Etgen, 2006).

Therapeutic regimens to recover from ischemic injuries are still scarce (Attari et al., 2016; Girbovan, Kent, Merali, \& Plamondon, 2016). Recent studies have focused on discovering new agents that can enhance hippocampal neurogenesis. These agents should possess antioxidant, antiapoptotic, or neuroprotective properties to overcome or limit the consequences of neural damage, and conse- quently prevent long-term cognitive impairments after brain ischemia (Attari et al., 2016; Gulinello et al., 2006).

Herbal medications and their active ingredients are a potential source for finding new remedies to improve cognitive and neurological deficits that occur following brain ischemia. Several studies supported the antiinflammatory, antioxidant, and neuroprotective effects of curcumin as an active constituent of turmeric (Shah et al., 2016). Because of its biological properties, curcumin is an appropriate candidate for improving injuries after brain ischemia. Previous studies support the beneficial effects of curcumin on neurogenesis, neuronal differentiation, neuroprotection, and improvement of cerebral pathological changes following brain ischemia (Attari et al., 2016; Liu et al., 2016; Shah et al., 2016; Zhao et al., 2010). However, only a few studies have reported the effects of curcumin on neurological and cognitive impairments after focal brain ischemia (Tu et al., 2014; Zhao et al., 2010) without mentioning GCI.

Besides, the earlier studies have mainly focused on evaluating the acute effects of curcumin, i.e. immedi- 
ately or up to 7 days after the induction of brain ischemia. Thus, there is no study on the long-term effects of treatment with curcumin and its time course effects on neurological and memory impairments following focal or GCI. Also, neuronal death in the CA1 area of the hippocampus has been already attributed to cognitive impairments after GCI (Wang, Xue, Zhao, Gao, \& Wei, 2012), but there is still no study on evaluating the neuronal aggregation following brain ischemia and the effect of curcumin treatment. Voronoi tessellation is a systematic way to characterize cell aggregation and provides information about the spatial distribution of cells in a specific area (Duyckaerts \& Godefroy, 2000; Jiao, Berman, Kiehl, \& Torquato, 2011).

The objectives of this study were three-fold. First to examine the time-course effects of long-term treatment with curcumin on the neurological symptoms and memory impairment, to evaluate the spatial distribution of the neurons in CA1 area following GCI, and finally to assess the acute and chronic effects of curcumin on the spatial neuronal distribution in CA1 area all after GCI.

\section{Methods}

\subsection{Animals}

A total of 56 adult male Sprague Dawley rats (weight: 200-250 g) were prepared from Shiraz University of Medical Sciences. The rats were kept under a 12:12 h light: dark cycle at $22 \pm 2^{\circ} \mathrm{C}$ with ad libitum feeding. Rats were acclimatized to their new conditions for one week before the study. All study procedures were done following the National Institute of Health guideline (1985).

\subsection{Study design}

The Sprague-Dawley rats were randomly assigned into four groups of sham $(n=14)$, control $(n=14)$, curcumin $50 \mathrm{mg} / \mathrm{kg}(\mathrm{n}=14)$, and curcumin $100 \mathrm{mg} / \mathrm{kg}(\mathrm{n}=14)$. Each group was subdivided into two subgroups of short-term ( 7 days) and long-term ( 28 days) treatment periods. Two treatment groups were given curcumin (Merck, Germany) orally dissolved in Phosphate Buffered Saline (PBS). The control group received $4 \mathrm{~mL} / \mathrm{kg}$ PBS orally, and the sham group received nothing. A single daily dose was administered (16-24 hours after operation) for 7 days in the short-term subgroup and 28 days in the long-term treatment subgroup. The curcumin dosage was determined based on a pilot study and previously published works (Liu et al., 2014).

\subsection{Induction of global cerebral ischemia}

Transient GCI was induced by using the two-vessel occlusion model (Kakkar, Muppu, Chopra, \& Kaur, 2013). After 12 hours of fasting, the rats were anesthetized using $100 \mathrm{mg} / \mathrm{kg}$ ketamine and $10 \mathrm{mg} / \mathrm{kg}$ xylazine; and two common carotid arteries were ligated using small artery clips for $20 \mathrm{~min}$ after separating the vagus nerve. The ischemia duration was chosen based on a pilot study and previous works. After $20 \mathrm{~min}$, the clips were removed from both common carotid arteries, and restoration of blood flow was visually confirmed. The rats whose pupils were not dilated and responsive to light and showed seizures were excluded from the experiments (Kakkar et al., 2013). In sham-operated rats, anesthesia and operations were performed, but common carotid arteries were not clamped.

\subsection{Assessment of neurological symptoms}

The Neurological Severity Score (NSS) was evaluated according to a previously described stroke index as follows: No neurological impairment: 0 ; Hunched posture: 1; Ptosis: 2; Circling behavior: 3; Splayed-out hind limb: 4; and Epileptic seizures: 5. The highest score indicates the most serious damage (Dai et al., 2013). The NSSs were assessed 6 hours after completing perfusion (postoperative day 0 ) and then, on postoperative days 1, 3, and 7 for short-term subgroup and days 1, 3, 7, 14, 21, and 28 for long-term treatment subgroup (Dong et al., 2016). On the first postoperative day (16-24 hours after operation), drug administration was begun, and evaluations were performed before, 1 and 3 hours after administration.

\subsection{Traction test}

Locomotor function was evaluated by the traction test, 15 minutes before step-through inhibitory avoidance task. Traction apparatus was a metallic wire $(2 \mathrm{~mm}$ diameter, $60 \mathrm{~cm}$ length) stretched horizontally between two wooden vertical planes $50 \mathrm{~cm}$ above the floor. The rats were first trained to remain on the wire for at least $180 \mathrm{~s}$. The time a rat took to remain suspended on the wire was recorded on postoperative days 1,3 , and 7 for short-term treatment subgroup and on days 1, 3, 7, 14, 21, and 28 for long-term treatment subgroup (Dong et al., 2016; Ahmadi-Mahmoodabadi, Nasehi, Emam-Ghoreishi, \& Zarrindast, 2016).

\subsection{Step-through inhibitory avoidance task}

The 2-way shuttle box (BorjSanaat Co., Tehran, Iran) consisted of two light (white opaque plexiglas, $20 \times 20 \times 30$ 
$\mathrm{cm}^{3}$ ) and dark (black opaque plexiglas, $20 \times 20 \times 30 \mathrm{~cm}^{3}$ ) compartments connected to each other by a sliding door $\left(8 \times 8 \mathrm{~cm}^{2}\right)$. Foot shock (Intensity: $0.7 \mathrm{~mA}$; Frequency: 50 $\mathrm{Hz}$; Duration: $3 \mathrm{~s}$ ) was delivered through rods of stainless steel grids on the floor of the dark compartment.

In the habituation trial, the rats were gently placed in the light compartment. 15 seconds later, the sliding door was opened to let the animals enter the dark compartment. When the rat completely entered the dark compartment, the sliding door was closed, and the rat was immediately returned to its home cage. Animals that waited for more than $120 \mathrm{~s}$ to enter into the dark compartment were excluded from the experiment. Thirty minutes later, the acquisition trial was performed. In this stage, when the rat entered into the dark compartment, the sliding door was closed, and a foot shock was instantly delivered to the grid floor of the dark compartment. After $15 \mathrm{~s}$, the rat was temporarily returned to its cage and tested again two minutes later. In case of entering the dark compartment, foot shock is given for the second time. The maximum training trial for each rat was three times. The retention or retrieval test was performed 24 hours later in a manner like the acquisition trial, but the electrical shock was not delivered to the grid floor. The time to enter the dark room (Step-through Latency (STL)) was recorded as an inhibitory avoidance memory. A cutoff time of $300 \mathrm{~s}$ for STL measurement was set for all animals that remained in the light room. The STL time was recorded on postoperative days 1,3 , and 7 for short-term treatment subgroup and on days 1, 3, 7, 14, 21, and 28 for long-term treatment subgroup (Ahmadi-Mahmoodabadi, Nasehi, Ghoreishi, \& Zarrindast, 2016).

\subsection{Novel object recognition test}

Recognition memory was assessed by the Novel Object Recognition Test (NORT) (Noorafshan, Karimi, Kamali, Karbalay-Doust, \& Nami, 2017). On days 5 and 26, after the operation, the rats in the 7-day and 28-day treatment subgroups were habituated respectively for $20 \mathrm{~min}$ in an empty white box with no roof $\left(100 \times 100 \times 40 \mathrm{~cm}^{3}\right)$. The following day, two similar colored cubes made of compact plastic were placed on the box floor, and rats were allowed to explore the objects for 3 min (the training phase). To evaluate the short-term memory, we replaced a new metallic gray cylinder $(5 \mathrm{~cm}$ in diameter and 10 $\mathrm{cm}$ in height) with one of the colored cubes 19 minutes later. Then, the rats were allowed to explore it for $3 \mathrm{~min}$. 24 hours later (day 7 or day 28 after operation) and 2 hours after the traction test, the long-term memory was evaluated by replacing one of the familiar objects with a novel object different from the previous one, letting rats explore it for $3 \mathrm{~min}$. In all phases, the total time that rats spent with the novel and familiar objects were recorded. The preference for exploration of novel objects was used as an index for a successful recognition memory test. The Novelty Preference Index (NPI) was calculated as follows: (time spent exploring the novel object - time spent exploring the familiar object)/ (time spent exploring the novel object + time spent exploring the familiar object).

\subsection{Preparation of brain sections}

After performing behavioral tests on days 7 and 28 after the operation, the rats' brains in the short-term and long-term treatment groups were fixed by transcardial perfusion of $0.1 \mathrm{M}$ PBS ( $\mathrm{pH} 7.4$ ) followed by $4 \%$ paraformaldehyde in PBS. Brains were removed and postfixed overnight and then transferred to $30 \%$ sucrose solution in $0.1 \mathrm{M} \mathrm{PBS}$ and kept at $4^{\circ} \mathrm{C}$ for $48-72$ hours (Dai et al., 2013). The samples were then kept at $-80^{\circ} \mathrm{C}$. $40 \mu \mathrm{m}$ thick coronal sections of the brains were cut by Leica CM1860 cryostat (Leica Microsystems, Solms, Germany) and stored in a cryoprotectant solution at $-20^{\circ} \mathrm{C}$ (Larsson, Lindvall, \& Kokaia, 2001) until using for cresyl violet staining. The staining was performed according to the protocol provided by the manufacturer (Merck) with minor modifications. The slides were then placed in the following solutions consecutively, 95\% alcohol for $1 \mathrm{~min}, 70 \%$ alcohol for $3 \mathrm{~min}$, distilled water for $5 \mathrm{~min}$, cresyl violet for $40 \mathrm{~min}$, distilled water for 3 $\mathrm{min}, 70 \%$ alcohol for $3 \mathrm{~min}, 95 \%$ alcohol for $5 \mathrm{~s}, 100 \%$ alcohol for $5 \mathrm{~s}$, butanol I for $2 \mathrm{~min}$, butanol II for $2 \mathrm{~min}$, xylol I for $2 \mathrm{~min}$, and xylol II for $2 \mathrm{~min}$. Afterward, the sections were cover-slipped with entlan glue.

\subsection{Evaluation of spatial distribution of neurons}

The spatial distribution of neurons in the CA1 region of the hippocampus was evaluated with the Voronoi tessellation method, which was obtained by constructing Voronoi polygons. Each polygon encompasses the areas in which the cells are accumulated and close together (Torquato, 2013). Thus, a polygon area indicates the spaces that a cell occupies. The area and the number of closest Voronoi polygons to each other were then obtained (Jiao et al., 2011). To draw the Voronoi polygon diagram, brain sections were analyzed using the videomicroscopy system. The CA1 microscopic images were taken with a 40x to1800x objective lens. The favored parameters were the neuron's nuclei. Each image was imported to the ImageJ software. After setting the scale of the images, we marked the neurons by clicking on their nuclei. In the next step, the polygons were designed 
by running "Plugins" then "Analyze" and finally "Voronoi" orders. Black and white images were taken from the tessellated areas with changing the threshold. Finally, the areas of polygons were measured by selecting "Analyze" and then "Measure" (Safaeian \& David, 2013; van Horssen et al., 2014).

\subsection{Statistical analysis}

The obtained data are presented as the Mean \pm SEM. The Kolmogorov-Smirnov test was performed to determine the normality of data distribution. Differences in NSS, STL time, and latency to fall time were assessed by repeated measures ANOVA by considering the time as a within-subjects factor and the treatment as a between-subjects factor. The least significant difference test was used as a post hoc test for comparing the mean differences between groups. If there was an interaction between time and treatment, 1-way ANOVA followed by Tukey's post hoc test were performed to evaluate the mean differences between groups at each time. The NORT data were analyzed by 1-way ANOVA, followed by Tukey's post hoc test for comparing means between groups. The approximate power of each analysis was computed with alpha level of 0.05 . The statistical analyses were performed in SPSS V. 21 (SPSS Inc., Chicago,
Illinois, USA). The statistical significance level was set as $\mathrm{P}<0.05$.

\section{Results}

\subsection{Effect of curcumin on neurological severity disorder}

Since the sham group had no neurological deficit (score: 0 ) throughout the study period, it was excluded from the statistical analysis. In the 7-day treatment subgroup, repeated measures ANOVA indicated the significant effect of time $(\mathrm{P}<0.001)$, no significant interaction between time and treatment, and a trend toward the significant impact of treatment $(\mathrm{P}=0.088)$. However, pairwise comparisons of means showed a significant difference between curcumin $100 \mathrm{mg} / \mathrm{kg}$ and control groups $(\mathrm{P}=0.039)$. Post hoc tests revealed a significantly lower NSS in curcumin $100 \mathrm{mg} / \mathrm{kg}$ group compared with the control group at postoperative day $7(\mathrm{P}<0.05)$ (Figure $1 \mathrm{~A})$.

In the 28-day treatment subgroup, repeated measures ANOVA results demonstrated a significant effect of time $(\mathrm{P}<0.001)$, treatment $(\mathrm{P}<0.001)$, and time $\times$ treatment interaction $(\mathrm{P}<0.001)$. Post hoc tests indicated a significant reduction in NSS for the curcumin $100 \mathrm{mg} /$ kg group compared with the control and curcumin 50 $\mathrm{mg} / \mathrm{kg}$ groups on postoperative days $7,14,21$, and 28

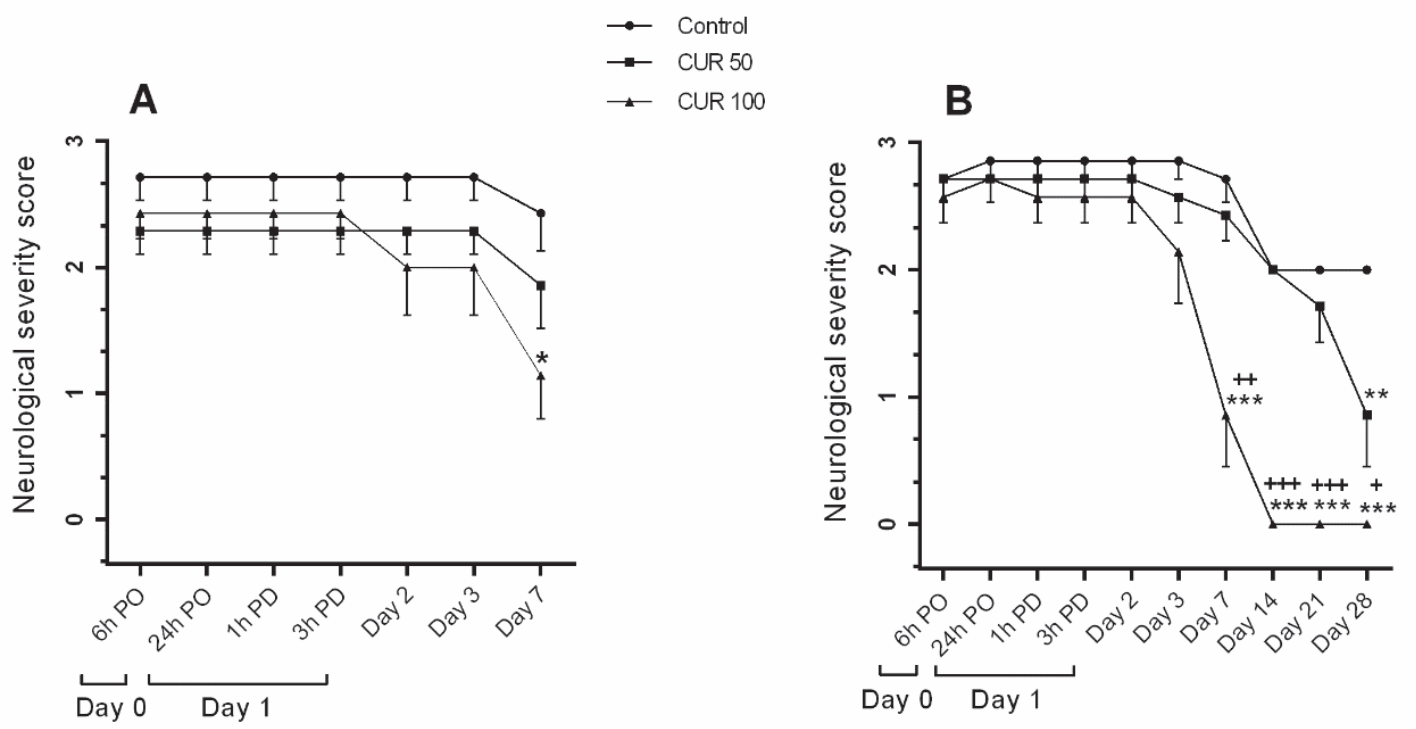

Figure 1. Data represent Mean \pm SEM of NSS in rats received vehicle (control)

A. Curcumin $50 \mathrm{mg} / \mathrm{kg}$ (CUR50) or curcumin $100 \mathrm{mg} / \mathrm{kg}$ (CUR100) for 7; B. 28 days after GCI. The NSS weUR evalugCIENCE different postoperative (PO) days i.e. day 0 (6 h PO), day 1 (24 h PO, 1 h after drug administration [PD] and 3 PD), days 2, 3 , $7,14,21$, and $28 \mathrm{PO}$

${ }^{*} \mathrm{P}<0.05 ; * * \mathrm{P}<0.01 ;{ }^{* * *} \mathrm{P}<0.001$ significantly different from the control group

$+\mathrm{P}<0.05 ;++\mathrm{P}<0.01 ;+++\mathrm{P}<0.001$ significantly different from CUR50 


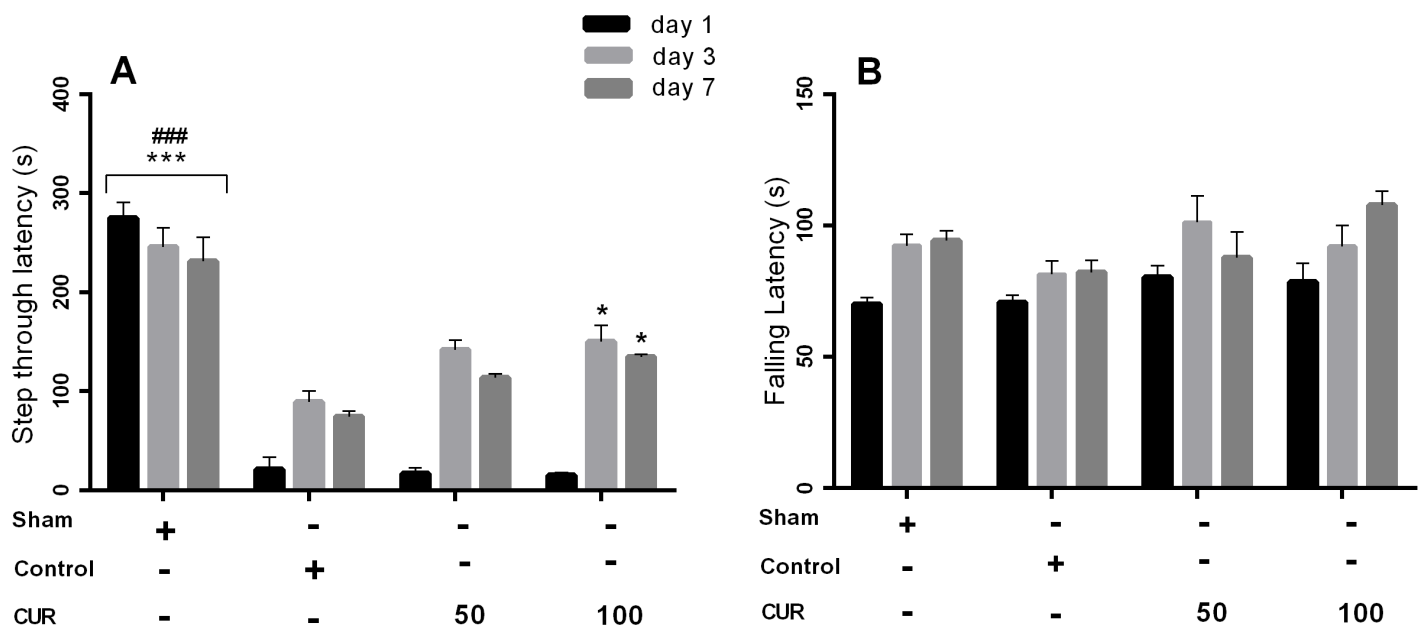

Figure 2. Bars represent Mean \pm SEM

NEUR OSCIENCE

A. STL time; B. Falling latency time (s) at days 1, 3, and 7 after GIC in the sham group and in rats received vehicle (control), curcumin $50 \mathrm{mg} / \mathrm{kg}$ (CUR50), or curcumin $100 \mathrm{mg} / \mathrm{kg}$ (CUR100) for 7 days

${ }^{*} \mathrm{P}<0.05 ; * * * \mathrm{P}<0.001$ significantly different from the control group

\#\#\#P<0.001 significantly different from control, CUR50, CUR100

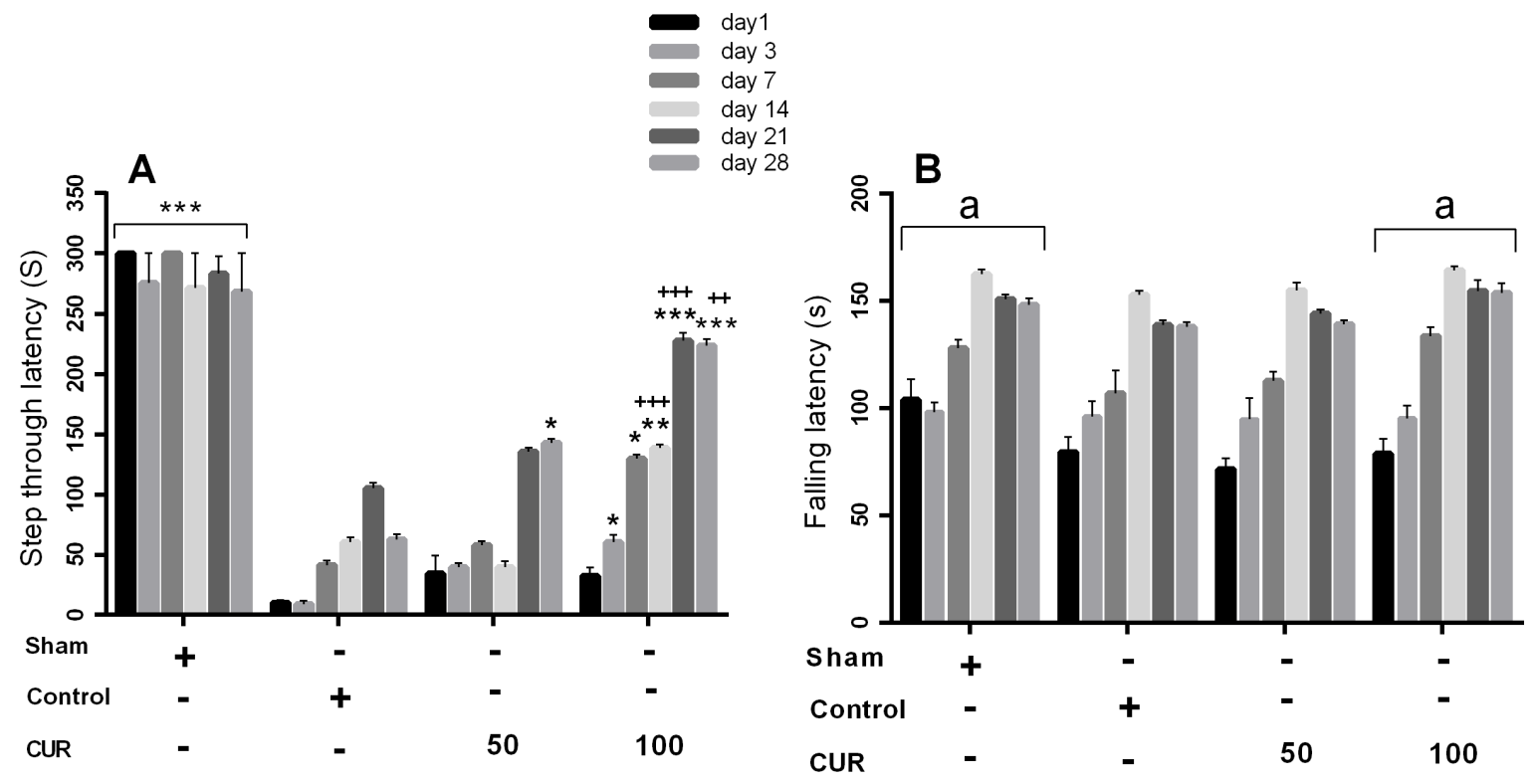

Figure 3. Bars represent Mean \pm SEM

NEUR SCIENCE

A. STL time; B. Falling latency time (s) at days 1, 3, 7, 14, 21, and 28 following GCI in sham group and in rats received vehicle (control), curcumin $50 \mathrm{mg} / \mathrm{kg}$ (CUR50), or curcumin $100 \mathrm{mg} / \mathrm{kg}$ (CUR100) for 28 days

${ }^{*} \mathrm{P}<0.05 ;{ }^{* *} \mathrm{P}<0.01 ; * * * \mathrm{P}<0.001$ significantly different from the control group

$+\mathrm{P}<0.05 ;++\mathrm{P}<0.01 ;+++\mathrm{P}<0.001$ significantly different from CUR50

a $\mathrm{P}<0.01$ : The mean of total latency to fall time throughout the study period significantly different from the control group 

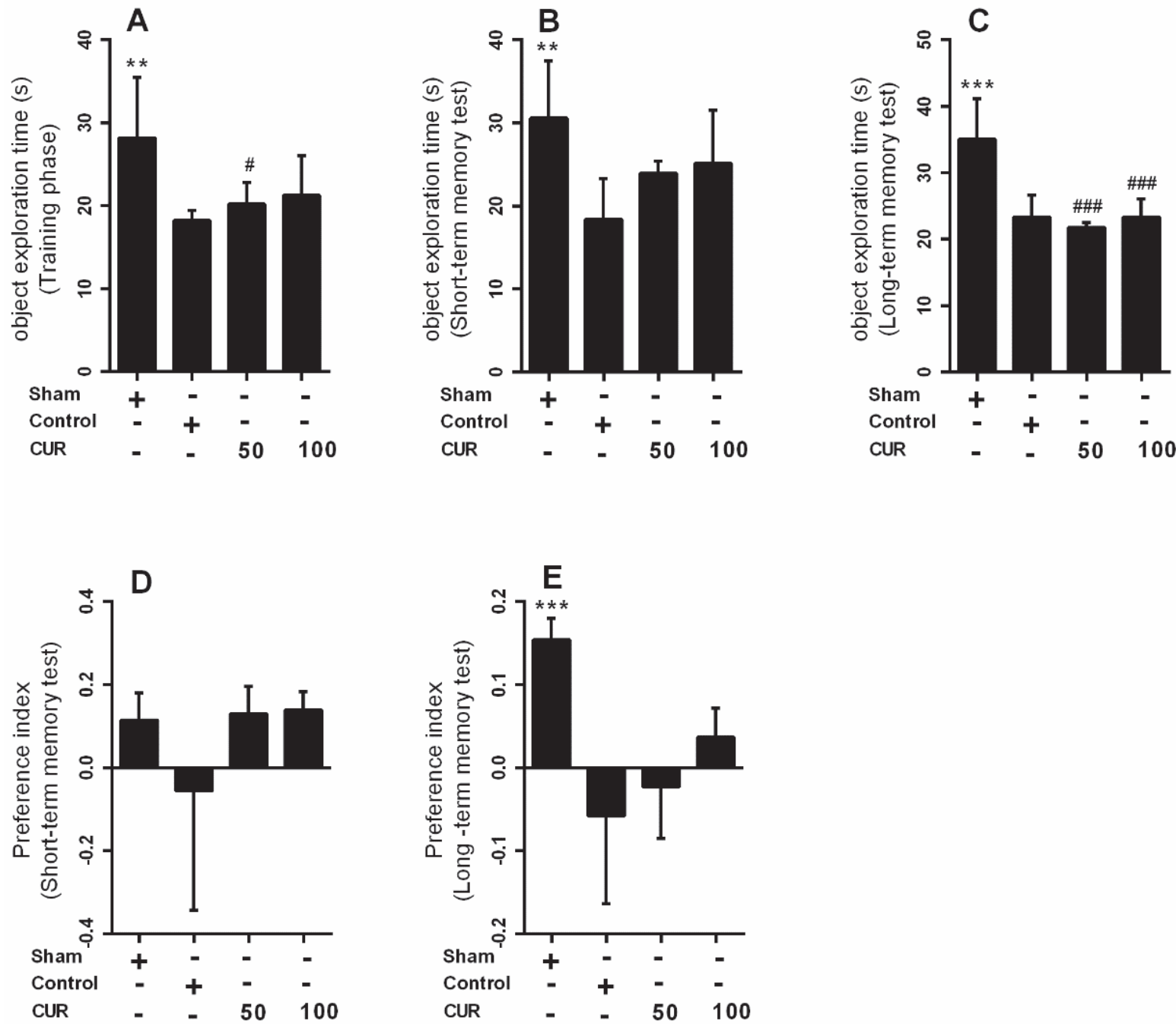

Figure 4. Box plots represent Mean \pm SEM of exploration times ( $n=7 /$ group)

NEUR:SCIENCE

A. The novel objects in training; B. Short-term; C. Long-term memory; D. Phases, novelty preference index for short-term; E. Long-term memory in NORT for the sham group and in rats received vehicle (control), curcumin $50 \mathrm{mg} / \mathrm{kg}$ (CUR50), or curcumin $100 \mathrm{mg} / \mathrm{kg}$ (CUR100) for 7 days after GCI

${ }^{*} \mathrm{P}<0.05$; ${ }^{* *} \mathrm{P}<0.01$; ${ }^{* * *} \mathrm{P}<0.001$ significantly different from the control group

$\# \mathrm{P}<0.05$; \#\#P<0.01; \#\#\#P<0.001 significantly different from the sham group

$(\mathrm{P}<0.05-\mathrm{P}<0.001)$ (Figure 1B). There was also a significantly lower NSS in $50 \mathrm{mg} / \mathrm{kg}$ curcumin-treated group on the postoperative day 28 compared with the control group $(\mathrm{P}<0.01)$ (Figure 1B).

\subsection{Effect of curcumin on passive avoidance task} and locomotor function

Repeated measures ANOVA showed the significant effect of time $(\mathrm{P}<0.001)$, treatment $(\mathrm{P}<0.001)$, and time $\times$ treatment interaction $(\mathrm{P}<0.001)$ on STL time in the 7-day treatment subgroup. The groups of control, curcumin $50 \mathrm{mg} / \mathrm{kg}$, and curcumin $100 \mathrm{mg} / \mathrm{kg}$, had significantly shorter STL times compared with the sham group at all study days $(\mathrm{P}<0.001)$. However, the STL time was significantly higher in curcumin $100 \mathrm{mg} / \mathrm{kg}$ group compared with the controls on postoperative days 3 and $7(\mathrm{P}<0.05)$ (Figure 2A). Repeated measures ANOVA showed a significant effect of time $(\mathrm{P}<0.001)$, but the treatment and time $\times$ treatment interaction had a significant impact on 

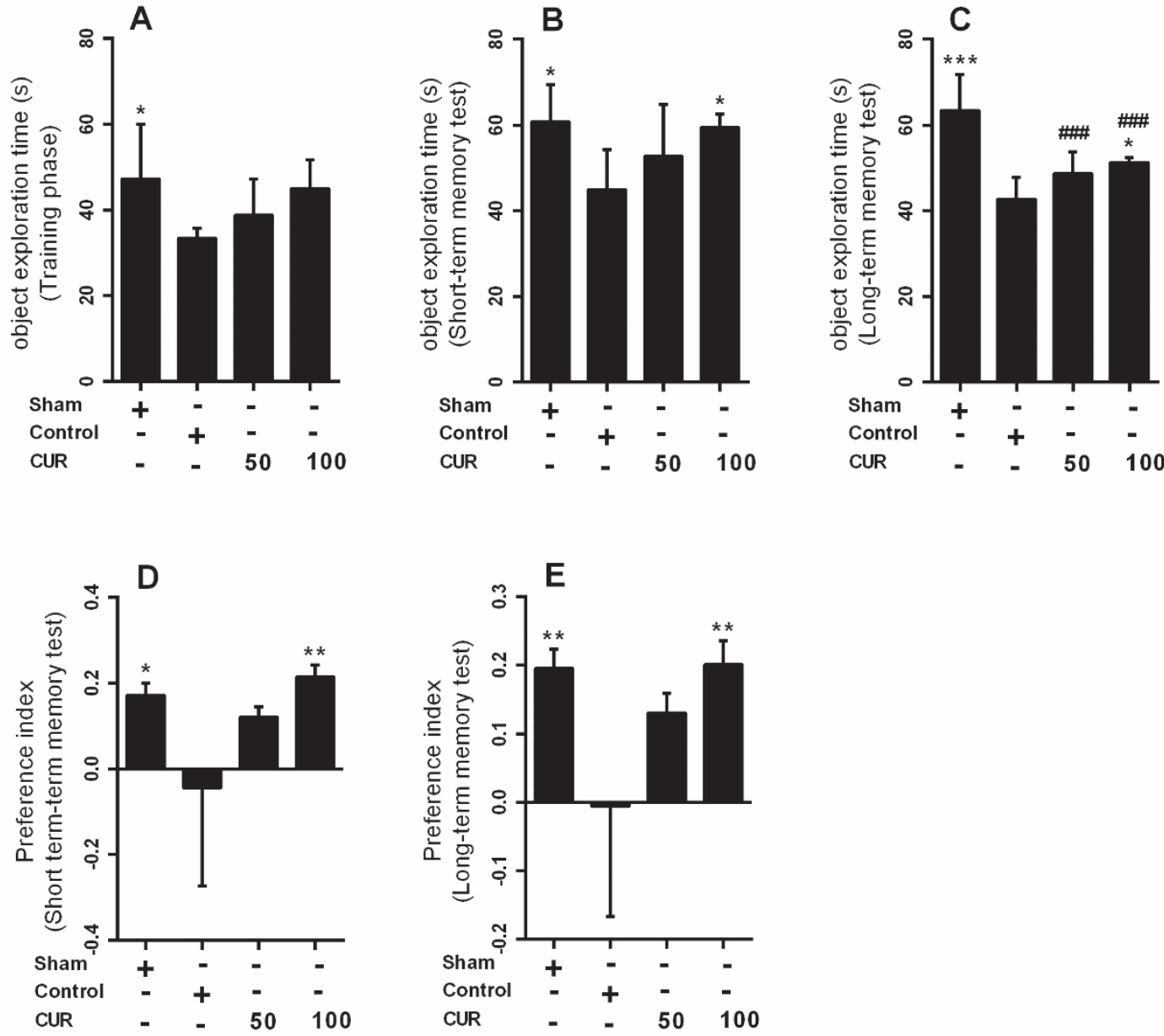

Figure 5. Box plots represent Mean \pm SEM of exploration times ( $n=7 /$ group)

NEUR:SCIENCE

A. The novel objects in training; B. Short-term; C. Long-term memory; D. Phases, novelty preference index for short-term; E Long-term memory in NORT for the sham group and rats received vehicle (control), curcumin $50 \mathrm{mg} / \mathrm{kg}$ (CUR50), or curcumin $100 \mathrm{mg} / \mathrm{kg}$ (CUR100) for 28 days after GCI

${ }^{*} \mathrm{P}<0.05 ; * * \mathrm{P}<0.01 ; * * \mathrm{P}<0.001$ significantly different from the control group

$\# \mathrm{P}<0.05 ; \# \# \mathrm{P}<0.01 ; \# \# \# \mathrm{P}<0.001$ significantly different from the sham group

latency to fall times (Figure 2B). The latency to fall time significantly increased on postoperative days 3 and 7 following operation compared with the time recorded on the first postoperative day $(\mathrm{P}<0.001)$.

In the 28-day treatment subgroup, repeated measures ANOVA results showed a significant effect of time $(\mathrm{P}<0.001)$, treatment $(\mathrm{P}<0.001)$, and time $\times$ treatment interaction $(\mathrm{P}<0.01)$ on $\mathrm{STL}$ time. Post hoc comparisons at each studied time revealed that the curcumin $100 \mathrm{mg} /$ $\mathrm{kg}$ group had significantly longer STL times on days 3 $(\mathrm{P}<0.05), 7(\mathrm{P}<0.05), 14(\mathrm{P}<0.01), 21(\mathrm{P}<0.001)$ and 28 $(\mathrm{P}<0.001)$ compared with the control group and on days
$14(\mathrm{P}<0.001), 21(\mathrm{P}<0.001)$, and $28(\mathrm{P}=0.01)$ compared with the curcumin $50 \mathrm{mg} / \mathrm{kg}$ group (Figure $3 \mathrm{~A}$ ). Besides, the STL time was significantly longer in the group treated with curcumin $50 \mathrm{mg} / \mathrm{kg}$ compared with the control group on days $21(\mathrm{P}<0.05)$ and $28(\mathrm{P}=0.01)$ (Figure $3 \mathrm{~A})$. The sham group showed significantly longer STL time compared with the samples in the groups of curcumin 50 $\mathrm{mg} / \mathrm{kg}$, curcumin $100 \mathrm{mg} / \mathrm{kg}$, and control on days $1,3,7$, 14 , and 21 after the operation $(\mathrm{P}<0.001)$. On day 28 , after the operation, the sham group showed a significantly longer STL time compared with the curcumin $50 \mathrm{mg} / \mathrm{kg}$ and control groups $(\mathrm{P}<0.001)$. 


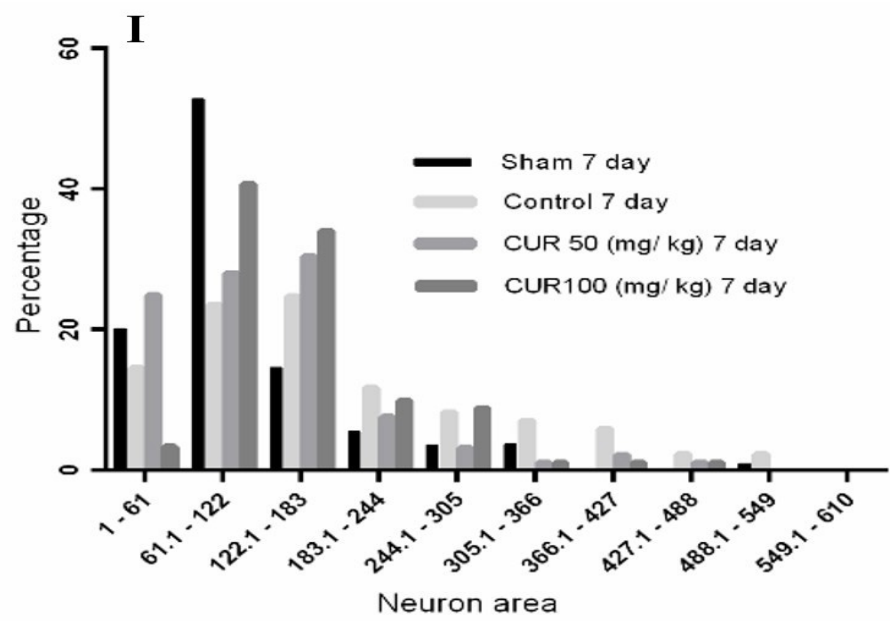

NEUR:SCIENCE

Figure 6. A micrograph of neurons in the CA1 for the groups

A. Sham; C. Control; E. Curcumin $50 \mathrm{mg} / \mathrm{kg}$ (CUR50); G. Curcumin $100 \mathrm{mg} / \mathrm{kg}$ (CUR100) in the 7-day treatment subgroups

obtained from Voronoi tessellation method. After setting the scale, neuronal nuclei were marked, and the polygons were superimposed

on them using the ImageJ software (B, D, F, H, respectively).

Lower bar graph (I) indicates the percentage of polygon distribution classified into various categories according to the polygon areas $(\mu \mathrm{m} 2)$

In the 28-day treatment subgroup, the results showed the significant effects of time $(\mathrm{P}<0.001)$ and treatment $(\mathrm{P}<0.01)$, but the time $\times$ treatment interaction had no significant effect on the latency to fall time. Significantly longer times for latency to fall were observed in the sham and curcumin $100 \mathrm{mg} / \mathrm{kg}$ groups compared with the controls $(\mathrm{P}<0.01)$ and curcumin $50 \mathrm{mg} / \mathrm{kg}$ group $(\mathrm{P}<0.01)$. Furthermore, the latency to fall times significantly increased on days 3 to 28 compared with the first day $(\mathrm{P}<0.001)$ and decreased on days 21 and 28 compared with day $14(\mathrm{P}<0.001)$.

\subsection{Effect of curcumin on the novel object recog- nition test}

In the 7-day treatment subgroup, there were no significant differences between NPI of the study groups in long-term memory test (Figure 4D); however, there was a significant reduction in NPI value in the control $(\mathrm{P}<0.001)$ and curcumin $50 \mathrm{mg} / \mathrm{kg}(\mathrm{P}<0.01)$ groups compared with the sham group in long-term memory test (Figure 4E).

In the 28-day treatment subgroup, the NPI in shortterm $(\mathrm{P}<0.05)$ and long-term memory $(\mathrm{P}<0.01)$ tests were significantly lower in the control group than in the sham group (Figure 5D and 5E). Moreover, the curcumin $100 \mathrm{mg} / \mathrm{kg}$ significantly increased the NPI in short- and long-term memory tests compared with the control group $(\mathrm{P}<0.01)$ (Figure $5 \mathrm{D}$ and $5 \mathrm{E})$. Linear regression analysis showed that the effect of curcumin on NPI in short-term (Mean $\pm \mathrm{SEM}=0.129 \pm 0.038 ; \mathrm{P}=0.003)$ and long-term memory $(\mathrm{Mean} \pm \mathrm{SEM}=0.103 \pm 0.03 ; \mathrm{P}=0.003)$ was dose-dependent; i.e. an increase in the curcumin dosage increases its effect on the recognition memory.

\subsection{Effect of curcumin on the spatial distribution of neurons in the CA1 area}

Figure 6 shows the Voronoi polygon areas calculated for the CA1 neuron's nuclei in the 7-day treatment subgroup. In the sham group, approximately $52.7 \%$ of the polygon areas were within the range of $61-122 \mu \mathrm{m}^{2}$, while $23.5 \%, 28 \%$, and $40.7 \%$ of the polygon areas ranged between 61 and $122 \mu \mathrm{m}^{2}$ in the control, curcumin $50 \mathrm{mg} / \mathrm{kg}$, and curcumin $100 \mathrm{mg} / \mathrm{kg}$ groups, respectively. Moreover, $20.1 \%$ and $27.2 \%$ of polygons in the sham group were stretched out within the range of 1-61 $\mu \mathrm{m}^{2}$ and 122-610 $\mu^{2}$, respectively. The areas of polygons within the range of $1-61 \mu \mathrm{m}^{2}$ were $14.6 \%, 25 \%$, and $3.4 \%$; and within the range of $122-610 \mu \mathrm{m}^{2}$ were $61.9 \%$, $47 \%$ and $55.9 \%$ for control, curcumin $50 \mathrm{mg} / \mathrm{kg}$ and curcumin $100 \mathrm{mg} / \mathrm{kg}$ groups, respectively.

In the 28-day treatment subgroup, $50.6 \%, 27.3 \%$, $36.7 \%$, and $44.7 \%$ of the polygons areas were found 
A

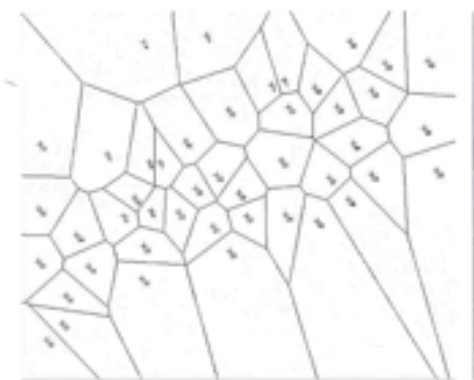

C

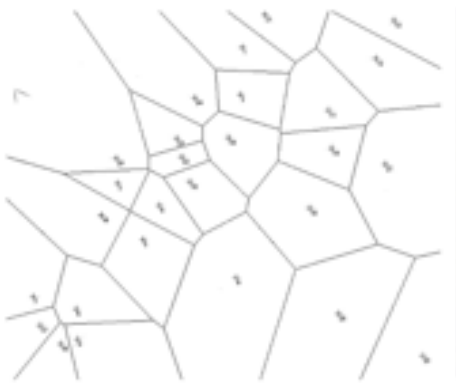

E

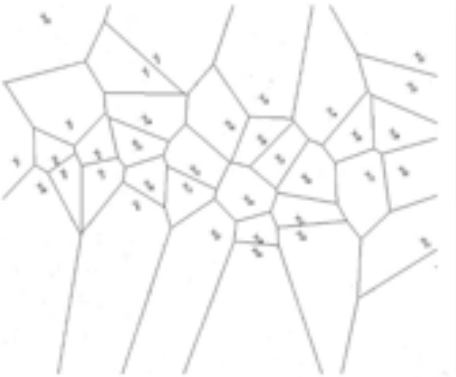

G

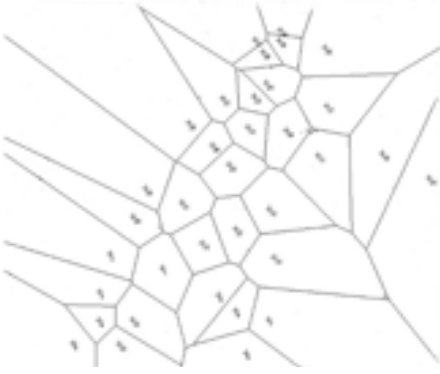

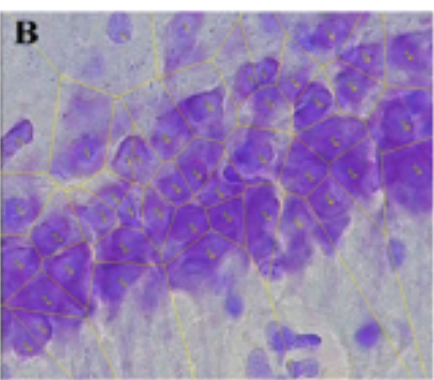
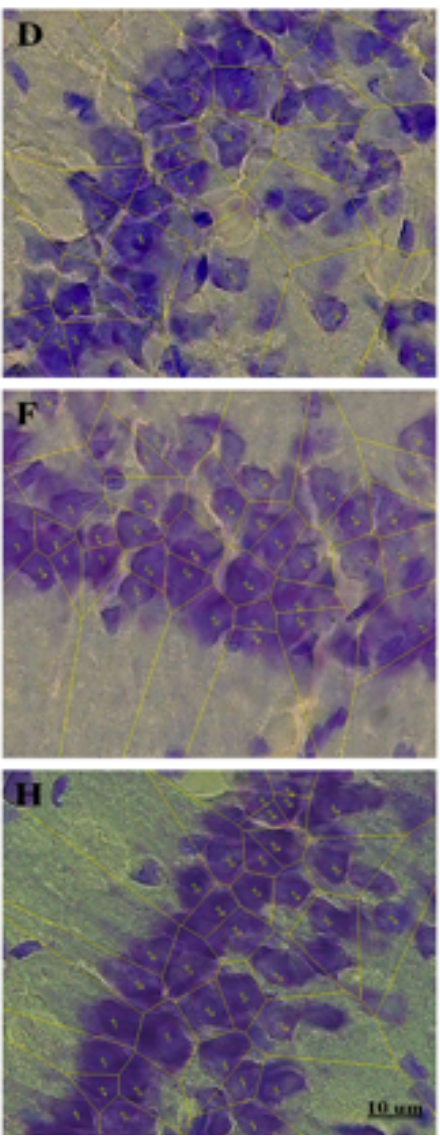

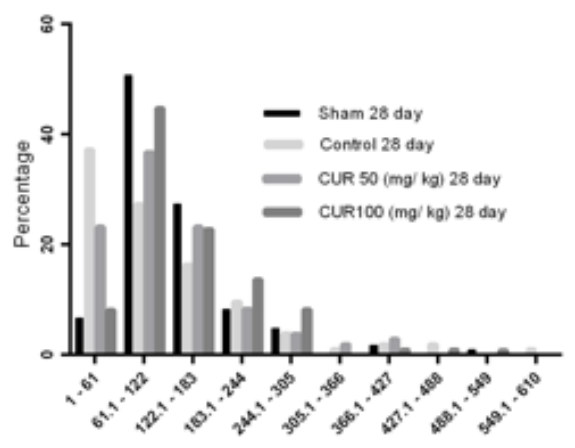

Neuron area

NEUROSCIENCE

Figure 7. A micrograph of neurons in the CA1 for the groups

A. Sham; C. Control; E. Curcumin 50 mg/kg (CUR50); G. Curcumin 100 mg/kg (CUR100) in the 7-day treatment subgroups obtained from Voronoi tessellation method. After setting the scale, neuronal nuclei were marked, and the polygons were superimposed on them using the ImageJ software (B, D, F, H, respectively).

Lower bar graph (I) indicates the percentage of polygon distribution classified into various categories according to the polygon areas $\left(\mu \mathrm{m}^{2}\right)$ 
within the range of $61-122 \mu \mathrm{m}^{2}$ in the sham, control, curcumin $50 \mathrm{mg} / \mathrm{kg}$ and curcumin $100 \mathrm{mg} / \mathrm{kg}$ groups, respectively. Moreover, the percentages of polygon areas for the sham, control, curcumin $50 \mathrm{mg} / \mathrm{kg}$ and curcumin $100 \mathrm{mg} / \mathrm{kg}$ groups were $6.6 \%, 37.2 \%, 23.3 \%$, and $8.1 \%$, within the range of $1-61 \mu^{2}$ and $42.8 \%, 35.5 \%, 40 \%$, and $47.2 \%$ within the range of $122-610 \mu \mathrm{m}^{2}$, respectively (Figure 7 A-I).

\section{Discussion}

The present study revealed that curcumin could improve neurological deficit and memory impairment and protected the normal distribution of neurons in the hippocampal CA1 region after GCI in a time-dependent manner. The beneficial effects of curcumin mainly started on day 7 after GCI and reached its highest level on day 28 post GCI. With a higher dosage $(100 \mathrm{mg} / \mathrm{kg})$, curcumin was more effective than with the lower dosage.

The results indicate that curcumin improves neurological deficits following GCI. A high dose of curcumin is more effective in improving neurological symptoms; i.e. it could completely recover neurological injury and accelerate the recovery period. To the best of our knowledge, there is no other time-course study on the effect of curcumin on neurological symptoms after GCI to be compared with our results. However, there is a report that curcumin with $100 \mathrm{mg} / \mathrm{kg}$ dose (not $50 \mathrm{mg} / \mathrm{kg}$ dose) could significantly reduce NSS 24 hours after focal ischemia (Yang, Zhang, Fan, \& Liu, 2009). In our study, on the contrary, $100 \mathrm{mg} / \mathrm{kg}$ curcumin could not significantly decrease NSS 24 hours after GCI. This discrepancy may be due to the difference in the used models of ischemia (global vs. focal ischemia).

Curcumin could improve memory after GCI, with a higher dose exerting better efficacy on memory restoration than its lower dose. Moreover, curcumin, even at a higher dose, could not completely revive impaired memory after GCI, because its maximum effect was not comparable to that of the sham group. To the best our knowledge, there is no other study on the impact of curcumin on memory impairment after GCI; however, previous reports have indicated that curcumin can improve memory in sleep-deprived rats (Noorafshan, Karimi, KarbalayDoust, \& Kamali, 2017), Alzheimer mice (Pan, Qiu, Lu, \& Dong, 2008), rats with colchicine-induced cognitive impairment (Kumar, Naidu, Seghal, \& Padi, 2007), and rates with 3-nitropropionic acid-induced cognitive impairment (Kumar, Padi, Naidu, \& Kumar, 2007). These reports agree with our study results that curcumin can ameliorate memory impairment following GCI.
The improved avoidance memory observed after using curcumin does not seem to be because of the effect of curcumin on locomotor function. Improved motor function over time in all study groups might indicate that the rats learned how to maintain their balance on the wire. Moreover, their overall motor function following GCI had improved by curcumin with a near-normal level. The reported time-course effects of curcumin on motor function agree with the improved NSSs, suggesting that the curcumin effect on motor function mirrored the recovery from the neurological deficit, but it had no direct effect on motor function. This finding is supported by the previous studies reported that curcumin could not affect locomotor activity (Zhao et al., 2012; Zhu et al., 2014). In any case, the improved memory observed after treatment with curcumin cannot be related to the effect of curcumin on locomotor function.

In this study, the improving effect of curcumin on memory was further supported by the findings that longterm treatment with curcumin can fully restore recognition memory following GCI. We found no study that has evaluated the effect of curcumin on the impaired recognition memory following GCI; however, consistent with our findings, some studies have reported that 21-day administration of curcumin can improve memory acquisition in aged male mice (Woolley, Marsden, Sleight, $\&$ Fone, 2003) and sleep-deprived rats subjected to the novel object-recognition task (Noorafshan et al., 2017).

In our study, the tessellation diagrams illustrate that GCI can cause an irregular neuronal distribution and a large gap between neurons in the CA1 region within the range of 61-122 $\mu \mathrm{m}^{2}$. The decreased neuronal aggregation may be due to either neuronal death or inflammation and indicates the inability of auto-recovery processes in reinstating the normal distribution of neurons. Curcumin could preserve normal aggregation of neurons in the CA1 region, depending on its dose and treatment duration. The antioxidant, anti-inflammatory, or neuroprotective properties of curcumin have likely led to neuronal survival after GCI. We suggest that these mechanisms be addressed in future studies.

The exact mechanism of curcumin in improving memory and reducing neurological deficit following GCI is still unknown; however, the antioxidant, anti-inflammatory, neuroprotective, and neurogenesis properties of curcumin may contribute to its beneficial effects after GCI. Time-dependent effects of curcumin on memory and NSS suggest that curcumin can exert its effects possibly through adaptive mechanisms that need time, including neurogenesis, altering synaptic plasticity or gene 
expression. The curcumin likely improves memory after GCI by increasing neurogenesis. This notion is in agreement with a recent study that showed that long-term administration of curcumin promoted neurogenesis and improved cognition in aged rats (Dong et al., 2012). The neuroprotective effect of curcumin has been reported in focal ischemia (Li, Tan, Yu \& Gang , 201; Wang et al., 2012; Zhuang, Lin, Song, \& Li, 2009) and GCI (Huang et al., 2011; Attari et al., 2016). These reports, consistent with our findings that curcumin could maintain the normal neuronal distribution in the CA1 region after GCI, suggest that neuroprotective and neurogenesis properties of curcumin can be related to its improving effects on the neurological deficits and memory impairments following GCI. We recommend that these mechanisms be addressed in future studies.

Curcumin improves memory function and neurological deficits, and maintain regular neuronal aggregation following GCI in a time-dependent manner. A high dose of curcumin $(100 \mathrm{mg} / \mathrm{kg})$ and its long-term administration (28 days) showed a greater effect on reviving GCI-induced impairments. Curcumin can be a potential candidate for improving memory and neurological problems after GCI.

\section{Ethical Considerations}

\section{Compliance with ethical guidelines}

The local Ethics Committee of Shiraz University of Medical Sciences and approved the study (Ethical code: IR.SUMS.REC.1395.S541).

\section{Funding}

This study was extracted from a $\mathrm{PhD}$. thesis in Neuroscience authored by Leila Kamali Dolatabadi. The project was financially supported by Shiraz University of Medical Sciences (Grant No. 94-01-74-11046).

\section{Authors' contributions}

Conceptualization: Masoumeh Emamghoreishi, Lelia Kamali Dolatabadi: Mohammad Reza Namavar; Methodology: All authors; Investigation: Lelia Kamali Dolatabadi, Hamze Badeli Sarkala; Writing-original draft: Lelia Kamali Dolatabadi; Writing-review \& editing: Masoumeh Emamghoreishi, Lelia Kamali Dolatabadi, Mohammad Reza Namavar; Funding acquisition: Masoumeh Emamghoreishi; Resources: Masoumeh Emamghoreishi; Supervision: Masoumeh Emamghoreishi.

\section{Conflict of interest}

The authors declared no conflict of interest.

\section{Acknowledgments}

The authors would like to thank Ms. Fatemeh Pirsalami and Ms. Maryam Mojahed for their technical supports. The authors also thank Mr. H. Argasi for his valuable assistance in editing this manuscript.

\section{References}

Ahmadi-Mahmoodabadi, N., Nasehi, M., Emam-Ghoreishi, M. E., \& Zarrindast, M. R. (2016). Synergistic effect between prelimbic 5-HT3 and CB1 receptors on memory consolidation deficit in adult male Sprague-Dawley rats: An isobologram analysis. Neuroscience, 317, 173-83. [DOI:10.1016/j.neuroscii ence.2015.12.010] [PMID]

Attari, F., Sharifi, Z. N., Movassaghi, S., Aligholi, H., Alizamir, T., \& Hassanzadeh, G. (2016). Neuroprotective effects of curcumin against transient global ischemia are dose and area dependent. Archives of Neuroscience, 3(2), e32600. [DOI:10.5812/ archneurosci.32600]

Dai, J., Chen, L., Qiu, Y. M., Li, S. Q., Xiong, W. H., Yin, Y. H., et al (2013). Activations of GABAergic signaling, HSP70 and MAPK cascades are involved in baicalin's neuroprotection against gerbil global ischemia/reperfusion injury. Brain Research Bulletin, 90,1-9. [DOI:10.1016/j.brainresbull.2012.09.014] [PMID]

Dong, H., Ma, Y., Ren, Z., Xu, B., Zhang, Y., Chen, J., et al. (2016). Sigma-1 receptor modulates neuroinflammation after traumatic brain injury. Cellular and Molecular Neurobiology, 36(5), 639-45. [DOI:10.1007/s10571-015-0244-0] [PMID]

Dong, S., Zeng, Q., Mitchell, E. S., Xiu, J., Duan, Y., Li, C., et al (2012). Curcumin enhances neurogenesis and cognition in aged rats: Implications for transcriptional interactions related to growth and synaptic plasticity. PLOS One, 7(2), e31211. [DOI:10.1371/journal.pone.0031211] [PMID] [PMCID]

Duyckaerts, C., \& Godefroy, G. (2000). Voronoi tessellation to study the numerical density and the spatial distribution of neurones. Journal of Chemical Neuroanatomy, 20(1), 83-92. [DOI:10.1016/S0891-0618(00)00064-8]

Girbovan, C., Kent, P., Merali, Z., \& Plamondon, H. (2016) Dose-related effects of chronic resveratrol administration on neurogenesis, angiogenesis, and corticosterone secretion are associated with improved spatial memory retention following global cerebral ischemia. Nutritional Neuroscience, 19(8), 35268. [DOI:10.1179/1476830515Y.0000000020] [PMID]

Gulinello, M., Lebesgue, D., Jover-Mengual, T., Zukin, R. S., \& Etgen, A. M. (2006). Acute and chronic estradiol treatments reduce memory deficits induced by transient global ischemia in female rats. Hormones and Behavior, 49(2), 246-60. [DOI:10.1016/j.yhbeh.2005.07.010] [PMID] [PMCID]

Günther, R., Suhr, M., Koch, J. C., Bähr, M., Lingor, P., \& Tönges, L. (2012). Clinical testing and spinal cord removal in a mouse model for Amyotrophic Lateral Sclerosis (ALS). Journal of Visualized Experiments, 17(61), e3936. [DOI:10.3791/3936] [PMID] [PMCID]

Huang, S., Wang, B., Zhang, Z. Q., Meng, Z. Y., Cao, H., Lian, Q. Q., et al. (2011). [Effect of curcumin on the expression of high 
mobility group box 1 and apoptotic neurons in hippocampus after global cerebral ischemia reperfusion in rats (Chinese)]. Zhonghua yi xue za zhi, 91(19), 1340-3. [PMID]

Jiao, Y., Berman, H., Kiehl, T. R., \& Torquato, S. (2011). Spatial organization and correlations of cell nuclei in brain tumors. PlOS One, 6(11), e27323. [DOI:10.1371/journal.pone.0027323] [PMID] [PMCID]

Kakkar, V., Muppu, S. K., Chopra, K., \& Kaur, I. P. (2013). Curcumin loaded solid lipid nanoparticles: An efficient formulation approach for cerebral ischemic reperfusion injury in rats. European Journal of Pharmaceutics and Biopharmaceutics, 85(3), 339-45. [DOI:10.1016/j.ejpb.2013.02.005] [PMID]

Kiryk, A., Pluta, R., Figiel, I., Mikosz, M., Ulamek, M., Niewiadomska, G., et al. (2011). Transient brain ischemia due to cardiac arrest causes irreversible long-lasting cognitive injury. Behavioural Brain Research, 219(1), 1-7. [DOI:10.1016/j. bbr.2010.12.004] [PMID]

Kumar, A., Naidu, P., Seghal, N., \& Padi, S. (2007). Effect of curcumin on intracerebroventricular colchicine-induced cognitive impairment and oxidative stress in rats. Journal of Medicinal Food, 10(3), 486-94. [DOI:10.1089/jmf.2006.076] [PMID]

Kumar, P., Padi, S., Naidu, P., \& Kumar, A. (2007). Possible neuroprotective mechanisms of curcumin in attenuating 3-nitropropionic acid-induced neurotoxicity. Methods and Findings in Experimental and Clinical Pharmacology, 29(1), 19-26. [DOI:10.1358/mf.2007.29.1.1063492] [PMID]

Larsson, E., Lindvall, O., \& Kokaia, Z. (2001). Stereological assessment of vulnerability of immunocytochemically identified striatal and hippocampal neurons after global cerebral ischemia in rats. Brain Research, 913(2), 117-32. [DOI:10.1016/ S0006-8993(01)02762-7]

Li, J., Shalabi, A., Ji, F., \& Meng, L. (2017). Monitoring cerebral ischemia during carotid endarterectomy and stenting. Journal of Biomedical Research, 31(1), 11-6. [PMID] [PMCID]

Li, L. I., Tan, B. T., Yu, L. I., \& Gang, Y. U. (2013). Protective effect and its mechanism of curcumin on ischemia-reperfusion injury of cerebral cortex in rats. Medical Journal of Chinese People's Liberation Army, 38(3), 190-4.

Liu, L., Zhang, W., Wang, L., Li, Y., Tan, B., Lu, X., et al. (2014). Curcumin prevents cerebral ischemia reperfusion injury via increase of mitochondrial biogenesis. Neurochemical Research, 39(7), 1322-31. [DOI:10.1007/s11064-014-1315-1] [PMID]

Liu, S., Cao, Y., Qu, M., Zhang, Z., Feng, L., Ye, Z., et al. (2016) Curcumin protects against stroke and increases levels of notch intracellular domain. Neurological Research, 38(6), 553-9. [DOI:1 0.1080/01616412.2016.1187804] [PMID]

Noorafshan, A., Karimi, F., Kamali, A. M., Karbalay-Doust, S. \& Nami, M. (2017). Restorative effects of curcumin on sleepdeprivation induced memory impairments and structural changes of the hippocampus in a rat model. Life Sciences, 189, 63-70. [DOI:10.1016/j.lfs.2017.09.018] [PMID]

Noorafshan, A., Karimi, F., Karbalay-Doust, S., \& Kamali, A. M (2017). Using curcumin to prevent structural and behavioral changes of medial prefrontal cortex induced by sleep deprivation in rats. EXCLI Journal, 16, 510-20. [DOI:10.17179/ex7 cli2017-139] [PMID] [PMCID]

Pan, R., Qiu, S., Lu, D. x., \& Dong, J. (2008). Curcumin improves learning and memory ability and its neuroprotective mechanism in mice. Chinese Medical Journal, 121(9), 832-9. [DOI:10.1097/00029330-200805010-00015] [PMID]
Safaeian, N., \& David, T. (2013). A computational model of oxygen transport in the cerebrocapillary levels for normal and pathologic brain function. Journal of Cerebral Blood Flow E Metabolism, 33(10), 1633-41. [DOI:10.1038/jcbfm.2013.119] [PMID] [PMCID]

Shah, F. A., Gim, S. A., Sung, J. H., Jeon, S. J., Kim, M. O., \& Koh, P. O. (2016). Identification of proteins regulated by curcumin in cerebral ischemia. Journal of Surgical Research, 201(1), 141-8. [DOI:10.1016/j.jss.2015.10.025] [PMID]

Torquato, S. (2013). Random heterogeneous materials: Microstructure and macroscopic properties. Berlin: Springer Science \& Business Media.

Tu, X. K., Yang, W. Z., Chen, J. P., Chen, Y., Ouyang, L. Q., Xu, Y. C., et al. (2014). Curcumin inhibits TLR2/4-NF-kB signaling pathway and attenuates brain damage in permanent focal cerebral ischemia in rats. Inflammation, 37(5), 1544-51. [DOI:10.1007/s10753-014-9881-6] [PMID]

van Horssen, P., van den Wijngaard, J. P., Brandt, M., Hoefer, I. E., Spaan, J. A., \& Siebes, M. (2014). Perfusion territories subtended by penetrating coronary arteries increase in size and decrease in number toward the subendocardium. American Journal of Physiology-Heart and Circulatory Physiology, 306(4), H496-H504. [DOI:10.1152/ajpheart.00584.2013] [PMID]

Wang, Z., Xue, R. L., Zhao, H. X., Gao, H., \& Wei, X. (2012). Effects of curcumin on neuronal apoptosis and neurobehavioral outcomes in rats after global cerebral ischemic and reperfusion injury. Practical Journal of Clinical Medicine, 6(011), 1-11.

Woolley, M. L., Marsden, C. A., Sleight, A. J., \& Fone, K. C. (2003). Reversal of a cholinergic-induced deficit in a roden model of recognition memory by the selective 5-HT6 receptor antagonist, Ro 04-6790. Psychopharmacology, 170(4), 358-67. [DOI:10.1007/s00213-003-1552-5] [PMID]

Yang, C., Zhang, X., Fan, H., \& Liu, Y. (2009). Curcumin upregulates transcription factor Nrf2, HO-1 expression and protects rat brains against focal ischemia. Brain research, 1282, 133-41. [DOI:10.1016/j.brainres.2009.05.009] [PMID]

Zhao, C., Lou, Z., Zimmer, B., Yu, Z., Li, P., Ma, B., et al. (2012). Chronic treatment with curcumin enhances methamphetamine locomotor sensitization and cue-induced reinstatement of methamphetamine self-administration. Pharmacology Biochemistry and Behavior, 102(4), 555-61. [DOI:10.1016/j. pbb.2012.06.011] [PMID]

Zhao, J., Yu, S., Zheng, W., Feng, G., Luo, G., Wang, L., et al (2010). Curcumin improves outcomes and attenuates focal cerebral ischemic injury via antiapoptotic mechanisms in rats. Neurochemical Research, 35(3), 374-9. [DOI:10.1007/s11064-0090065-y] [PMID]

Zhu, Q., Sun, Y., Yun, X., Ou, Y., Zhang, W., \& Li, J. X. (2014). Antinociceptive effects of curcumin in a rat model of postoperative pain. Scientific Reports, 4, 4932. [DOI:10.1038/srep04932] [PMID] [PMCID]

Zhuang, R., Lin, M., Song, Q., \& Li, J. (2009). [Effects of curcumin on the expression of nuclear factor-kappaB and intercellular adhesion molecular 1 in rats with cerebral ischemia-reperfusion injury (Chinese)]. Journal of Southern Medical University, 29(6), 1153-5. [PMID] 
This Page Intentionally Left Blank 